\title{
DESTAQUE TERRITORIAL: PROPOSTA DE MODELAGEM SOCIOECONÔMICA E AMBIENTAL PARA AVALIAR A INSERÇÃO SOCIAL NOS PROGRAMAS DE PÓS-GRADUAÇÃO EM CIÊNCIAS AMBIENTAIS
}

GEOGRAPHIC HIGHLIGHTS: A SOCIOECONOMIC \& ENVIRONMENTAL MODEL PROPOSED TO ASSESS THE SOCIAL INSERTION OF POSTGRADUATE PROGRAMS IN ENVIRONMENTAL SCIENCES

Rodrigo Affonso de

Albuquerque Nóbrega (D)

Coordenador no Programa de Pós-Graduação em Análise e Modelagem de Sistemas

Ambientais da Universidade Federal de Minas Gerais (UFMG) - Belo Horizonte (MG), Brasil.

Sónia Maria Carvalho Ribeiro (D) Professor titular no Programa de PósGraduação em Análise e Modelagem de Sistemas Ambientais da UFMG Belo Horizonte (MG), Brasil.

Elaine Lopes da Costa (D)

Discente no Programa de Pós-Graduação em Análise e Modelagem de Sistemas Ambientais da UFMG - Belo Horizonte (MG), Brasil.

\section{Patrícia Bilotta (D)}

Professora titular no Programa de Pós-

Graduação em Gestão Ambiental,

Universidade Positivo - Curitiba (PR), Brasil.

Isabel Jurema Grimm (D)

Coordenadora no Programa de

Pós-Graduação em Governança e

Sustentabilidade, Instituto Superior de Administração e Economia/Fundação Getulio Vargas - Curitiba (PR), Brasil.

Carlos Alberto Cioce Sampaio (D) Professor titular no Programa de Pós-Graduação em Desenvolvimento Regional, Universidade Regional de Blumenau (FURB) - Blumenau (SC), Brasil. Professor titular no Programa de Pós-Graduação em Gestão Ambiental da Universidade Positivo - Curitiba (PR), Brasil.

\section{Andreia Schypula (iD)}

Discente do Programa de Graduação em Engenharia Civil, Universidade Positivo Curitiba (PR), Brasil.

\section{Joselisa Maria Chaves (D)}

Professora titular no Programa de Pós-

Graduação em Modelagem em Ciências da Terra e do Ambiente, Universidade Estadual de Feira de Santana (UEFS) - Feira de Santana (BA), Brasil.

Washington de Jesus

Sant'Anna da Franca Rocha (D)

Professor titular no Programa de Pós-Graduação em Modelagem em Ciências da Terra e do Ambiente, UEFS - Feira de Santana (BA), Brasil.

Rodrigo Nogueira de Vasconcelos (D) Pesquisador no Programa de Pós-Graduação em Modelagem em Ciências da Terra e do Ambiente, UEFS - Feira de Santana (BA), Brasil.

Endereço para correspondência: Patrícia Bilotta - Rua Professor Pedro Viriato de Souza, 5.300 - Campo Comprido CEP 81280-330 - Curitiba (PR), Brasil E-mail:pb.bilotta@gmail.com

\section{RESUMO}

Este artigo propõe um método para mapear e qualificar a inserção social dos Programas de Pós-Graduação (PPG) na área de Ciências Ambientais da Coordenação de Aperfeiçoamento de Pessoal de Nível Superior (CAPES). A construção do modelo consistiu na integração espacial de três componentes: o índice de vulnerabilidade socioeconômica (infraestrutura, capital humano, renda e trabalho); o índice de hemerobia (grau de antropização no uso e na ocupação do solo); e a densidade geográfica dos PPG. O modelo utiliza álgebra de mapas para produzir o mapa de destaque territorial com índices variando entre 1 (baixo) e 10 (alto). A validação ocorreu na avaliação quadrienal 2017, quando consultores da CAPES utilizaram o mapa de destaque territorial para interpretar qualitativamente a inserção social dos PPG frente às vulnerabilidades socioeconômicas e ambientais regionais. Outras aplicações vislumbradas são: diagnóstico de regiões prioritárias para novos cursos, direcionamento de recursos (humano e financeiro), incentivo à criação de redes colaborativas e análise da inserção profissional dos egressos dos PPG.

Palavras-chave: vulnerabilidade; análise ecossocioeconômica; densidade geográfica.

\section{ABSTRACT}

This article proposes a method to map and qualify the social insertion of Postgraduate Programs in CAPES' Environmental Sciences area. The model was built based on the spatial integration of three key components: the socioeconomic vulnerability index (infrastructure, human capital, income and labor), the hemeroby index (land use and occupation), and the Postgraduate Programs' geographic density. The solution uses map algebra to output the Geographic Highlights, which indexes ranging from 1 (low) to 10 (high). The validation of the model occurred with the 2017 quadrennial evaluation, when Geographic Highlights map supported CAPES consultants to qualitatively interpret the social insertion of Postgraduate Programs in the face of regional socioeconomic and environmental vulnerabilities. Other applications envisaged are: identification of priority areas for new courses, resource allocation (human and financial), encouraging the creation of collaborative networks, and, finally, analysis of former students' professional insertion.

Keywords: vulnerability; ecosocioeconomic analysis; geographic density. 


\section{INTRODUÇÃO}

De acordo com a classificação da Coordenação de Aperfeiçoamento de Pessoal de Nível Superior (CAPES), a área de Ciências Ambientais está inserida na Grande Área Multidisciplinar, a qual, por sua vez, se enquadra no Colégio de Ciências Exatas, Tecnológicas e Multidisciplinar. A área reúne, atualmente, 123 Programas de Pós-Graduação (PPG), que equivalem a 154 cursos: 87 mestrados acadêmicos, 31 mestrados profissionais e 36 doutorados, distribuídos por todas as regiões geográficas do Brasil (CAPES, 2018).

Cada unidade da Federação possui programas da área de Ciências Ambientais e eles estão devidamente interiorizados, com exceção do Estado do Acre, onde a Coordenação de Área (mandato 2014-2018) induziu uma proposta de criação de curso de mestrado, que será apreciada pela Comissão de Avaliação das Propostas de Cursos Novos (APCN) em 2018 (SAMPAIO; CORTEZ; SCHIMITT, 2017).

As Ciências Ambientais, como área do conhecimento, contrapõem-se à fragmentação a que tem sido submetida a ciência e surgem como campo eminentemente interdisciplinar, uma vez que a problemática ambiental é indissociável dos sistemas sociais (SOBRAL et al., 2014; FERNANDES; SAMPAIO, 2016; ESTOQUE; MURAYAMA, 2017; KRAKER, 2017). Sob tal argumento, o quesito Inserção Social, um dos cinco preceitos do Sistema Nacional de Pós-Graduação (SNPG), poderia ser redenominado como Inserção Socioambiental. Os quesitos de avaliação dos Programas de Pós-Graduação são: 1. proposta do programa; 2 corpo docente; 3. corpo discente, teses e dissertações; 4. produção intelectual; e 5. inserção social

Cabe ressaltar que, na área de Ciências Ambientais, o quesito Inserção Social apresenta o maior peso permitido pela CAPES para o processo de avaliação de PPG $(20 \%$ para programas acadêmicos e $25 \%$ para profissionais), o que o torna um dos critérios mais significativos na área, em comparação com as demais 48 áreas de avaliação (SAMPAIO; CORTEZ; SCHIMITT, 2016). Destaca-se também que a finalidade desse quesito é avaliar o impacto socioambiental dos PPG, por consequência de suas pesquisas científicas, de modo que se possa aferir sua contribuição à sociedade (DONOVAN, 2008).

Segundo a Direção Geral de Pesquisa da Comissão Europeia, os impactos sociais podem repercutir positivamente, no sentido de promover qualidade de vida, estimular políticas públicas mais adequadas às demandas sociais, influenciar o desenvolvimento de novas abordagens e debates sobre assuntos de interesse social, encorajar mudanças coletivas de atitude, comunicar avanços no conhecimento, entre outras perspectivas (EUROPEAN COMISSION, 2010). Por isso, a mensuração e a avaliação do impacto social decorrente de investimentos financeiros e de recursos humanos aplicados em pesquisas acadêmicas têm sido objeto de vários estudos (DONOVAN, 2008; FURTADO et al., 2009; SPAAPEN; DROOGE, 2011; PENFIELD et al., 2014). Todavia, não há um consenso sobre a metodologia mais adequada a esse propósito (DONOVAN, 2008; REF, 2014) e alguns autores consideram essa uma difícil tarefa, pois há o risco de se criar mecanismos que interfiram na dinâmica da produção do conhecimento, por meio, por exemplo, da inserção de métricas que estimulam a competitividade em prejuízo da cooperação (DIAS SOBRINHO, 2004) e da pressão pelo cumprimento de critérios quantitativos em detrimento da qualidade da produção científica (SPAGNOLO; SOUZA, 2004).

Diante desse desafio, a coordenação da área de Ciências Ambientais tem buscado parâmetros consistentes que permitam sinalizar os impactos socioeconômicos e ambientais, designados ecossocioeconômicos, decorrentes das atividades dos PPG e das qualificações de sua atuação, sobretudo em áreas de fronteira, de interiorização e de vulnerabilidade social, econômica e ambiental (SAMPAIO; CORTEZ; SCHIMITT, 2017).

Como ponto de partida, propôs-se uma primeira versão sistematizada de avaliação, denominada de Destaque Territorial, concebida e implementada a partir da junção de três componentes:

1. o índice de vulnerabilidade socioeconômica, nas perspectivas de infraestrutura, capital humano, renda e trabalho (IPEA, 2015);

2. o índice de vulnerabilidade socioambiental, baseado na antropização da paisagem sintetizada pelo uso e pela cobertura do solo no território nacional (IBGE, 2012);

3. a concentração geográfica dos PPG em Ciências Ambientais. 
Nesse contexto, o objetivo deste artigo é analisar a inserção socioeconômica e ambiental dos programas cadastrados na área de Ciências Ambientais da CAPES com base nos parâmetros da metodologia proposta.

Este trabalho propõe uma metodologia espacialmente explícita desenvolvida com a finalidade de mapear o destaque territorial dos PPG na área de Ciências Ambientais da CAPES e fomentar o aperfeiçoamento da coordenação dos PPG quanto ao direcionamento de suas atividades de ensino, pesquisa e extensão para áreas prioritárias. 0 modelo foi implementado por meio de análise espacial das três dimensões mencionadas acima, recorrendo a técnicas de geoprocessamento e análise multicritério (NOBREGA, 2009). Para tanto, utilizou-se o método Analytical Hierarchy Process (AHP), proposto por Saaty (1994), em um ambiente de Sistema de Informação Geográfica (SIG), para aplicar a eficiência analítica do método de organização e tomada de decisão no contexto espacial (FERREIRA JUNIOR; NOBREGA; OLIVEIRA, 2016; NOBREGA, 2009; SADASIVUNI et al., 2009).

Este trabalho é o resultado de esforços colaborativos realizados por uma equipe de pesquisadores de três PPG da área de Ciências Ambientais, por solicitação de sua coordenação, no mandato anterior e no atual, com o intento de mapear as assimetrias regionais das dimensões socioeconômica e ambiental para, assim, aperfeiçoar o indicador Destaque Territorial, em conformidade com o Plano Nacional de Pós-Graduação 2011-2020 (BRASIL, 2010). O modelo produziu resultados concretos que fomentaram ações pioneiras de apoio à tomada de decisão na coordenação da área de Ciências Ambientais, bem como auxiliaram no processo de distribuição de bolsas no PPG Análise e Modelagem de Sistemas Ambientais (AMSA) da Universidade Federal de Minas Gerais (UFMG).

Contudo, embora o modelo esteja apoiado em um arcabouço robusto, composto de técnicas consolidadas de geoprocessamento e análise multicritério, os resultados podem ser aperfeiçoados, seja pela inclusão de outras variáveis, seja pela reorganização dos pesos atribuídos às variáveis, entre outras questões, a partir do modelo conceitual proposto e dos resultados deste estudo. Diante disso, uma segunda versão do método está em desenvolvimento, a fim de aprimorar o indicador Destaque Territorial, em razão dos limites do alcance de sua aferição, conforme se destaca nos Resultados e na Conclusão deste artigo.

\section{Geoprocessamento como suporte à tomada de decisão}

A complexidade das variáveis no processo de planejamento e gestão do território, bem como o volume de dados envolvidos têm sido fatores preponderantes no emprego de modelos computacionais para otimizar o processo de tomada de decisão (O'HARA; DAVIS; KLEISS, 2000). Com isso, há uma crescente demanda pela utilização de técnicas de geoprocessamento como chave para os sistemas de suporte à decisão espacial.

Entende-se como geoprocessamento o processo sistemático de empregar dados espaciais, ferramentas de análise, modelos e atores para computar informações quantitativas e/ou qualitativas que consideram os aspectos do contexto geográfico dos dados ou do fenômeno em análise (BURROUGH; MCDONNELL, 1998). Contudo, deve-se destacar que a grande contribuição do geoprocessamento é a sua capacidade de promover a análise espacial. Segundo Câmara et al. (2004), a análise espacial visa incorporar a componente espacial a uma análise com fim específico, quantificando propriedades e relacionando fenômenos em uma forma espacialmente explícita.
O uso do geoprocessamento auxilia na identificação da localização ("onde?") de um fenômeno, cuja resposta pode desencadear soluções importantes para o planejamento e a gestão do território (RYERSEN; ARONOFF, 2010). De forma análoga, responder a questões como "quando?" e "quanto?" qualifica a capacidade analítica da administração pública, na condição de formular cenários preditivos e quantificar impactos negativos e positivos oriundos desses cenários, além de subsidiar a formulação de políticas públicas (NOBREGA, 2018). A demanda por informações espaciais estruturadas foi uma das principais responsáveis pelos avanços conquistados pelos SIG nas últimas décadas. O estágio tecnológico atual, com a disseminação de dados geográficos e de ferramentas que operam em plataformas pessoais, tem permitido a consolidação do emprego do geoprocessamento no processo de tomada de decisão (STICH et al., 2011).

Segundo Nobrega (2018), embora as administrações municipais, estaduais e federal tenham consciência da 
necessidade de contemporizar o processo de formulação de argumentos para apoiar a correta tomada de decisão em planejamento e gestão territorial, o Estado ainda não se encontra aparelhado por instrumentos, modelos e recursos humanos adequados ao aprimoramento desse processo em muitos de seus setores (NOBREGA et al., 2012). Ferraz et al. (2015) ressaltam que, com a maturidade dos sistemas de geoprocessamento como ferramenta de apoio ao planejamento e à gestão territorial, o rigor da política ambiental e a crescente demanda pela participação pública nos processos decisórios, é inaceitável que a gestão pública esteja suscetível a repetir erros cometidos no passado, sem que se busquem soluções sistêmicas de como contorná-los (FERRAZ et al., 2015).

Nesse contexto, o geoprocessamento surge como uma ferramenta qualificada capaz de promover a organização, a integração e o controle das informações, das regras e dos atores envolvidos no processo de tomada de decisão espacial (SADASIVUNI et al., 2009). A disparidade entre os atores envolvidos, comumente defendendo interesses próprios, eleva o grau de complexidade requerido pelos processos de tomada de decisão (STICH et al., 2011) e, por isso, a necessidade de ferramentas robustas para garantir o processamento dos dados de forma transparente, quanto ao acesso às variáveis e regras de decisão, e replicáveis, quanto à sua capacidade de reproduzir ou aperfeiçoar o modelo.

A análise multicritério espacial é uma importante ferramenta na tomada de decisões estratégicas e tem se destacado tanto na iniciativa privada quanto na gestão governamental. Trabalhos recentes desenvolvidos para o Tribunal de Contas da União (TCU) comprovam a eficiência do modelo hierárquico de inteligência geográfica por análise multicritério para apoio estratégico à tomada de decisão (FERRAZ et al., 2015).

De forma análoga aos trabalhos conduzidos pelo TCU, o modelo proposto neste artigo recorre à metodologia AHP, implementada em ambiente SIG, com dados de entrada convertidos para o formato matricial (raster) e trabalhados por pixel. A arquitetura do modelo é hierárquica, planejada para decompor a solução do problema em etapas e atender a diferentes níveis de decisão.

\section{MATERIAIS E MÉTODOS}

O modelo proposto consistiu na integração espacial de três critérios distintos de análise:

- aspectos socioeconômicos;

- aspectos socioambientais;

- densidade geográfica (ou concentração) dos PPG.

\section{Bases de dados}

Para representar o critério socioeconômico, utilizou-se o Índice de Vulnerabilidade Social (IVS), proposto pelo Instituto de Pesquisas Econômicas Aplicadas (IPEA, 2015) e adotado internacionalmente (HUMMELL; CUTTER; EMRICH, 2016). O IVS é o resultado da média aritmética de três subíndices (Infraestrutura Urbana, Capital Humano e Renda e Trabalho), com igual peso, obtidos a partir de vários indicadores (Figura 1).

Para o critério socioambiental, foi empregado o nível de antropização da paisagem, por meio da aplicação do índice de hemerobia, por ser amplamente reportado em estimativas do nível de pressão antrópica no contexto da paisagem (BELEM; NUCCl, 2011;
Por ser um protótipo analítico-espacial, desenvolvido para dar suporte à tomada de decisão, a metodologia utilizada no modelo recorre a ferramentas e índices consagrados na literatura.

RÜDISSER; TASSER; TAPPEINER, 2012; WALZ; STEIN, 2014; WELLMANN et al., 2018).

Como ponto de partida, recorreu-se ao mapa de cobertura e uso do solo, disponibilizado pelo Instituto Brasileiro de Geografia e Estatística (IBGE, 2012), em escala 1:5.000.000, de todo o território nacional. No mapa, a superfície é classificada segundo diferentes tipologias, com base em informações adquiridas pelas equipes da coordenação de geociências do IBGE e no referencial internacional do programa de compilação de dados sobre uso e cobertura da Terra e monitoramento de mudanças ocorridas, da Agência Ambiental Europeia, denominado CORINE (AAE, 2018). 
Para o critério de isolamento geográfico, valeu-se da localização dos 123 PPG da área de Ciências Ambientais da CAPES e sobre eles aplicaram-se recursos de análise espacial para mapear sua densidade (ou concentração) no território nacional. Partiu-se da premissa de que esse critério permite mapear o isolamento geográfico

\section{Modelagem dos dados}

Embora simples, no que tange à quantidade de dados, o modelo proposto integra variáveis complexas, como a vulnerabilidade socioambiental e o isolamento geográfico, essenciais no diagnóstico das ações estratégicas da coordenação da área de Ciências Ambientais da

\section{Componente socioeconômico}

O componente socioeconômico, representado pelo IVS, é um índice calculado para o município e que dos PPG e das suas áreas de atuação, em termos de ações de ensino, pesquisa e extensão.

O Quadro 1 resume os dados de entrada, bem como as respectivas ferramentas e regras definidas para a modelagem espacial.

CAPES e dos PPG. Por isso, a integração entre os componentes considerados no estudo (Figura 2 ) requer a preparação das variáveis, incluindo a normalização dos valores e a álgebra de mapas para a composição dos cenários finais.

indica a exclusão e vulnerabilidade social identificadas no território, variando entre 0 e 1 . O IPEA

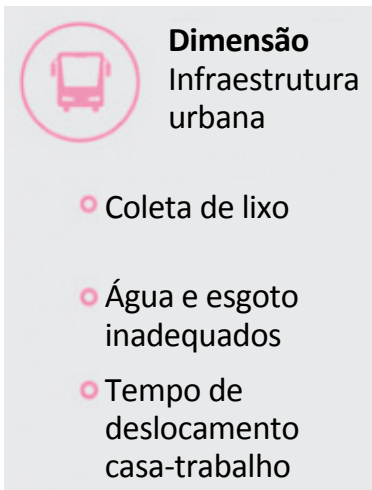

Dimensão Infraestrutura urbana casa-trabalho

\begin{tabular}{|c|c|c|}
\hline $\begin{array}{l}\text { Dimensão } \\
\text { Capital } \\
\text { humano }\end{array}$ & & $\begin{array}{l}\text { Dimensão } \\
\text { Renda e } \\
\text { trabalho }\end{array}$ \\
\hline $\begin{array}{l}\text { Mortalidade } \\
\text { infantil }\end{array}$ & Mães jovens (10 a 17 anos) & \multirow{2}{*}{$\begin{array}{l}\text { Renda menor ou igual a } \mathrm{R} \$ 255 \\
\text { Baixa renda e dependente } \\
\text { de idosos }\end{array}$} \\
\hline $\begin{array}{l}\text { Crianças de } 0 \text { a } \\
5 \text { anos fora da escola }\end{array}$ & $\begin{array}{l}\text { Mães sem ensino fundamental } \\
\text { + filhos até } 15 \text { anos }\end{array}$ & \\
\hline $\begin{array}{l}\text { Não estudam, não } \\
\text { trabalham e baixa renda }\end{array}$ & Analfabetismo & - Trabalho infantil \\
\hline $\begin{array}{l}\text { Crianças de } 6 \text { a } 14 \text { anos } \\
\text { fora da escola }\end{array}$ & $\begin{array}{l}\text { ninguém tem o ensino } \\
\text { fundamental completo }\end{array}$ & $\begin{array}{l}\text { Ocupação informal sem } \\
\text { ensino fundamental }\end{array}$ \\
\hline
\end{tabular}

Fonte: IPEA (2015).

Figura 1 - Dimensões consideradas na composição do Índice de Vulnerabilidade Social.

Quadro 1 - Fontes de dados utilizados na composição das variáveis do modelo.

\begin{tabular}{|c|c|c|c|}
\hline Dado & Fonte & Ferramenta adotada & Regra de modelagem \\
\hline $\begin{array}{l}\text { Localização dos Programas de Pós- } \\
\text { Graduação em Ciências Ambientais }\end{array}$ & $\begin{array}{l}\text { CAPES } \\
(2018)\end{array}$ & Densidade espacial & $\begin{array}{c}\text { Regiões distantes dos Programas de } \\
\text { Pós-Graduação apresentam maior } \\
\text { vulnerabilidade }\end{array}$ \\
\hline Mapa de uso e cobertura do solo & $\begin{array}{l}\text { IBGE } \\
(2012)\end{array}$ & $\begin{array}{l}\text { Reclassificação de } \\
\text { mapa temático }\end{array}$ & $\begin{array}{l}\text { Reclassificação do mapa de uso do solo } \\
\text { segundo o nível de antropização da } \\
\text { paisagem, usando o índice de hemerobia }\end{array}$ \\
\hline Índice de Vulnerabilidade Social & $\begin{array}{l}\text { IPEA } \\
(2015)\end{array}$ & $\begin{array}{l}\text { Integração dos dados } \\
\text { aos polígonos dos } \\
\text { municípios }\end{array}$ & $\begin{array}{l}\text { Reclassificação das categorias do } \\
\text { Índice de Vulnerabilidade Social }\end{array}$ \\
\hline
\end{tabular}


orienta a classificação do índice em cinco categorias: 0 a 0,200 - muito baixa vulnerabilidade social; 0,201 a 0,300 - baixa vulnerabilidade social; 0,301 a 0,400 - média vulnerabilidade social; 0,401 a 0,500 - alta vulnerabilidade social; e 0,501 a 1 - muito alta vulnerabilidade social (IPEA, 2015). O IVS é gerado por município e fornecido na forma de tabela, a qual contém uma coluna referente ao código municipal com sete dígitos, utilizado como chave para o relacionamento entre os dados da tabela e os

\section{Componente socioambiental}

Existe uma vasta literatura sobre índices socioambientais que analisam as dinâmicas na paisagem (FUSHITA, 2011; CARVALHO-RIBEIRO et al., 2016), porém os resultados dessas análises variam em escala, extensão e intensidade de acordo com a interação ou intervenção do polígonos dos municípios oriundos da base de dados territorial do IBGE.

De forma que promovam a normalização dos dados, os índices de vulnerabilidade foram agregados e reclassificados para o intervalo entre 1 e 10 . Os novos valores de IVS foram, então, atribuídos aos polígonos dos municípios, e o mapa resultante foi convertido do formato vetorial para o formato matricial. A resolução espacial adotada para a composição dos mapas em formato raster foi de $500 \mathrm{~m}$.

homem na natureza (HAFFER, 1992; LANG; BLASCHKE, 2009). Nesse sentido, o estudo da variação espacial em paisagem em diferentes escalas é atributo da Ecologia da Paisagem (Associação Internacional de Ecologia da Paisagem) (IALE, 1998).

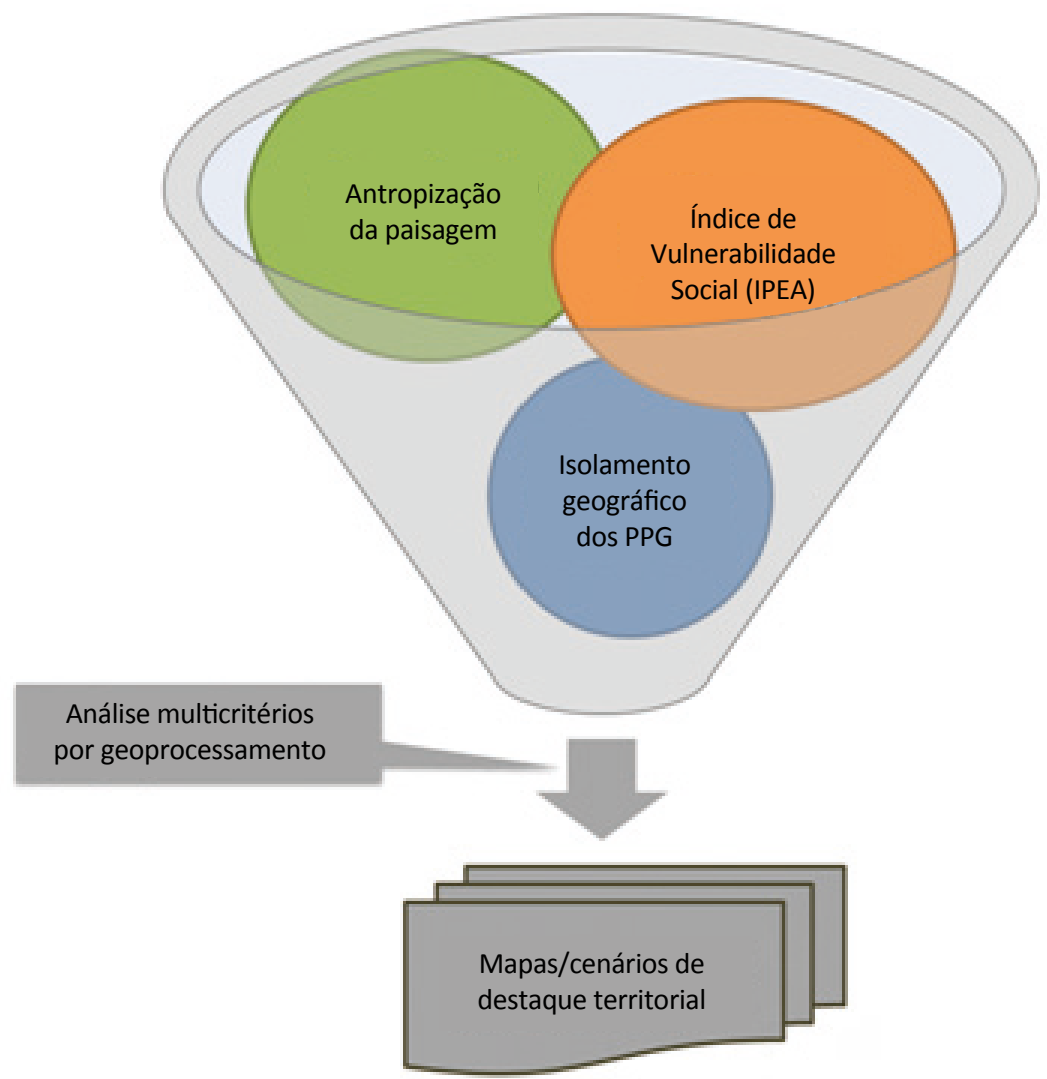

Figura 2 - Representação da integração dos componentes socioeconômico, socioambiental e geográfico para a construção do mapa de destaque territorial. 
Um dos índices utilizados na literatura internacional para medir alterações da paisagem ao longo do tempo é o índice de hemerobia, que mede o nível de antropização da paisagem. As alterações ocorridas na cobertura da terra podem ser classificadas em nenhuma alteração, onde a paisagem é considerada "natural" ou com pouca antropização (ahemerobiotico); "quase natural", com mais aspectos naturais do que artificiais (oligohemerobiotico); "semi (agri) natural", com mais aspectos artificiais do que naturais (mesohemerobiotico); e as "agri-cultural" que são artificiais (euhemerobiotico) (JALAS, 1953 apud TROPPMAIR, 1989; SUKOPP, 1972).

O índice de hemerobia é muito utilizado para associar classes de uso e cobertura da terra a níveis de antropização e intensificação do uso do solo em um dado momento no tempo (FUSHITA et al., 2017). Essa análise permite avaliar o grau de antropização de diferentes paisagens e traz grande relevância internacional, visto que a perda global de biodiversidade e serviços ecossistêmicos é, cada vez mais, associada a pressões antrópicas associadas ao uso e à cobertura do solo.

\section{Componente de isolamento geográfico}

A inclusão do componente espacial permitiu ao modelo considerar as regiões do Brasil não atendidas ou atendidas por poucos PPG na área de Ciências Ambientais da CAPES. A premissa é que a demanda por ações de ensino, pesquisa e extensão em regiões que não tenham tais PPG seja superior à demanda em regiões já amparadas pela CAPES.

Para o desenvolvimento do modelo, utilizou-se o operador Kernel, que estima a densidade espacial de ocorrência de um determinado evento (RYERSON; ARONOFF, 2010; BURROUGH; MCDONNELL, 1998). Esse operador gera uma superfície matricial de valores

\section{Análise multicritério}

A análise multicritério consiste em um procedimento utilizado para auxiliar a tomada de decisão em sistemas complexos (SAATY, 1994) e, quando combinada com as geotecnologias, permite reduzir o tempo de análise, além de ilustrar as regiões de maior e menor viabilidade de implantação e desenvolvimento de projetos na forma de mapas, gráficos e tabelas (STICH et al., 2011; NOBREGA, 2009; MALCZEWSKI, 1999).
O mapa dos índices de hemerobia gerado neste estudo foi construído a partir da associação entre os níveis de antropização do território brasileiro e as 31 classes de cobertura e uso do solo do IBGE (2012) reclassificadas para assumir valores entre 1 e 10 (Quadro 2). A nota máxima atribuída ao nível de antropização (10) foi designada às classes "floresta plantada" e "áreas urbanas". Essa premissa simplifica a realidade, por não diferenciar a composição e a estrutura tanto das florestas plantadas quanto das áreas urbanas. Seguindo o mesmo princípio, os menores níveis de antropização (1 e 2) foram atribuídos às classes "corpos de água" e "matas/florestas naturais" (Quadro 2).

Além da reclassificação, realizou-se a reamostragem espacial do dado matricial. De forma análoga à componente socioeconômica, o mapa de hemerobia foi compilado com a resolução espacial de $500 \mathrm{~m}$ para compatibilizar os componentes e possibilitar a análise multicritério.

de densidade, ao aplicar-se uma análise espacial de vizinhança circular, para computar as ocorrências dos pontos dentro do raio de busca. Os valores variaram entre 0 (ausência total de pontos) e 1 (presença máxima de pontos), para cada posição analisada (pixel), e, posteriormente, foram reclassificados para o intervalo entre 1 e 10, para compatibilizar o critério de densidade geográfica com os outros componentes do modelo. O processo foi desenvolvido na plataforma Esril ArcGIS e o raio definido para o operador Kernel foi a distância média calculada entre os PPG da área de Ciências Ambientais. A Figura 3 apresenta um recorte do mapa de densidade de PPG do modelo proposto.

Neste trabalho, empregou-se álgebra de mapas (O'HARA; DAVIS; KLEISS, 2000) para combinar as três dimensões consideradas (Figura 4) (Equação 1). A variação dos pesos atribuídos a cada critério (p1, p2 e p3) possibilitou o cálculo de diferentes cenários para a escolha da solução mais apropriada.

Destaque Territorial $=$ Socioambiental $* p 1$

+ Socioeconômico $* p 2+$ Geográfica $* p 3$ 
Quadro 2 - Reclassificação do uso do solo em classes de hemerobia.

1

2

3

4

5

6

7

8

9

10

11

12

13

14

15

16

17

18

19

20

21

22

23

24

25

26

27

28

29

30

31
Área entre 50 e $25 \%$ ocupada por estabelecimentos agropecuários

Área entre 25 e 10\% ocupada por estabelecimentos agropecuários Área com menos de $10 \%$ de ocupação por atividade agropecuária

Florestas plantadas

Área urbanizada

Pastagens + matas e/ou florestas

Pastagens

Lavouras + pastagens

Pastagens plantadas

Usos diversificados

Matas e/ou florestas + pastagens

Lavouras + matas e/ou florestas

Pastagens + lavouras

Lavouras temporárias

Matas e/ou florestas + lavouras

Lavouras

Outras coberturas e usos + usos diversificados

Matas e/ou florestas naturais

Pastagens naturais

Pastagens + sistemas agroflorestais

Lavouras permanentes

Sistemas agroflorestais + usos diversificados

Matas e/ou florestas

Pastagens + outras coberturas e usos

Outras coberturas e usos

Sistemas agroflorestais

Matas e/ou florestas + outras coberturas e usos

Uso de corpo d'água costeiro para pesca, aquicultura, lazer e turismo

Lavouras + outras coberturas e usos

Lavouras + sistemas agroflorestais

Matas e/ou florestas + sistemas agroflorestais
4

3

2

10

10

5

8

7

9

5

7

5

8

7

5

9

5

1

2

3

7

4

1

8

5

3

2

1

9

6

2 
O resultado do processo de álgebra de mapas é um dado matricial, em que os valores atribuídos aos pixels correspondem à grandeza do fenômeno em análise. $\mathrm{O}$ produto é comumente denominado como superfície de custo acumulado, pois os valores dos pixels representam o custo de oportunidade atribuído à posição geográfica de cada um deles. Quanto mais alto o valor, maior é o custo (ou esforço) a ser despendido para uma determinada ação, no contexto espacial do pixel em questão (NOBREGA, 2009; SADASIVUNI, 2009).

\section{RESULTADOS E DISCUSSÃO}

A modelagem espacial proposta utilizou três critérios distintos para a construção do mapa de destaque territorial e possibilitou a criação de cenários diversos, elaborados como alternativas para a "análise de sensibilidade"

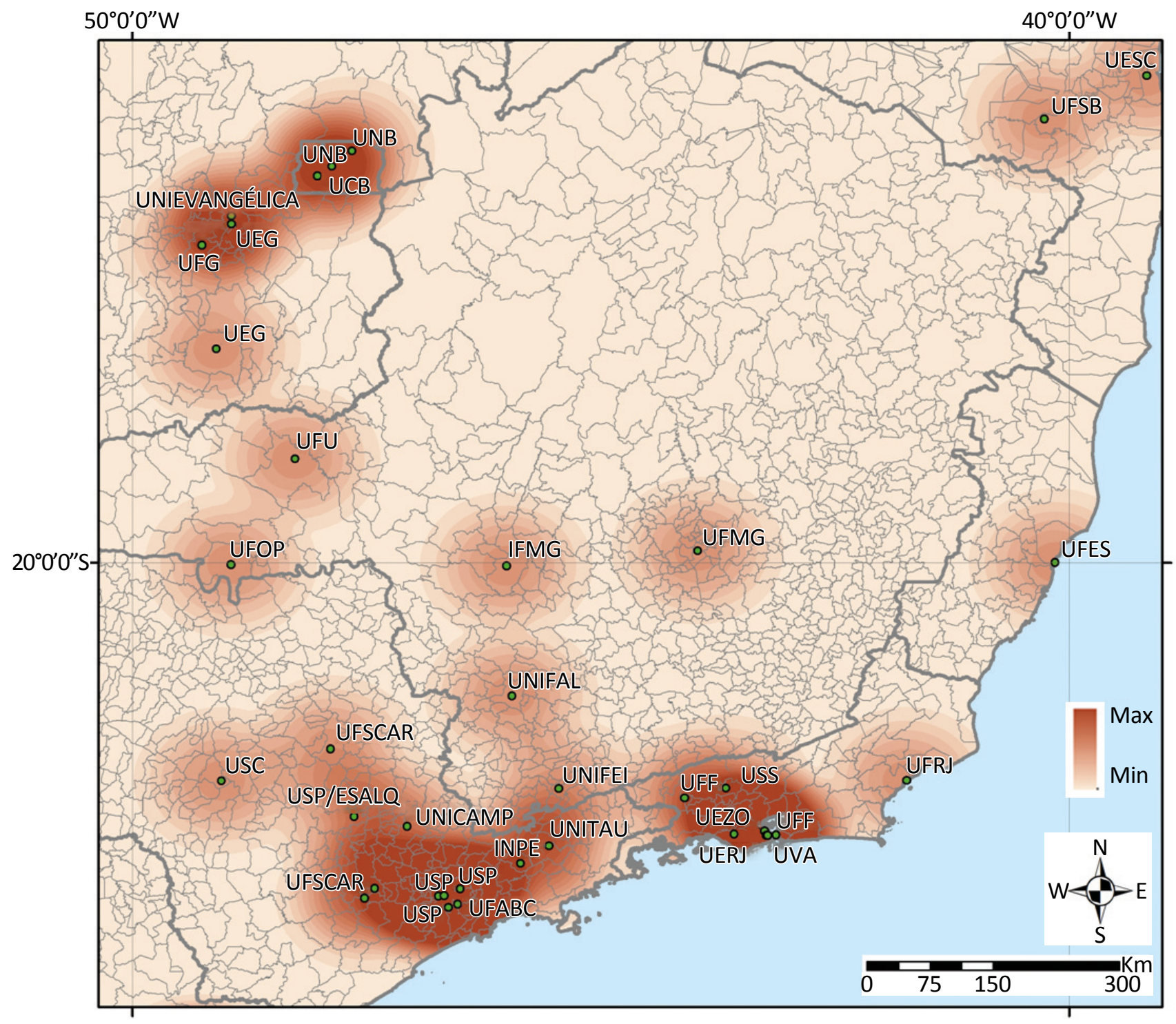

IPEA: Instituto de Pesquisa Econômica Aplicada PPG: Programas de Pós-Graduação

Figura 3 - Recorte do mapa de densidade dos Programas de Pós-Graduação nas Ciências Ambientais da Coordenação de Aperfeiçoamento de Pessoal de Nível Superior. 
aplicada na avaliação da inserção socioambiental dos PPG na área de Ciências Ambientais. A Figura 5 mostra os quatro cenários produzidos pela variação da atribuição de pesos (participação) das três dimensões consideradas.
O cenário 1 acentua a participação do critério socioambiental e, consequentemente, as regiões com índices de destaque territorial mais acentuados foram influenciadas pelo alto grau de antropização da

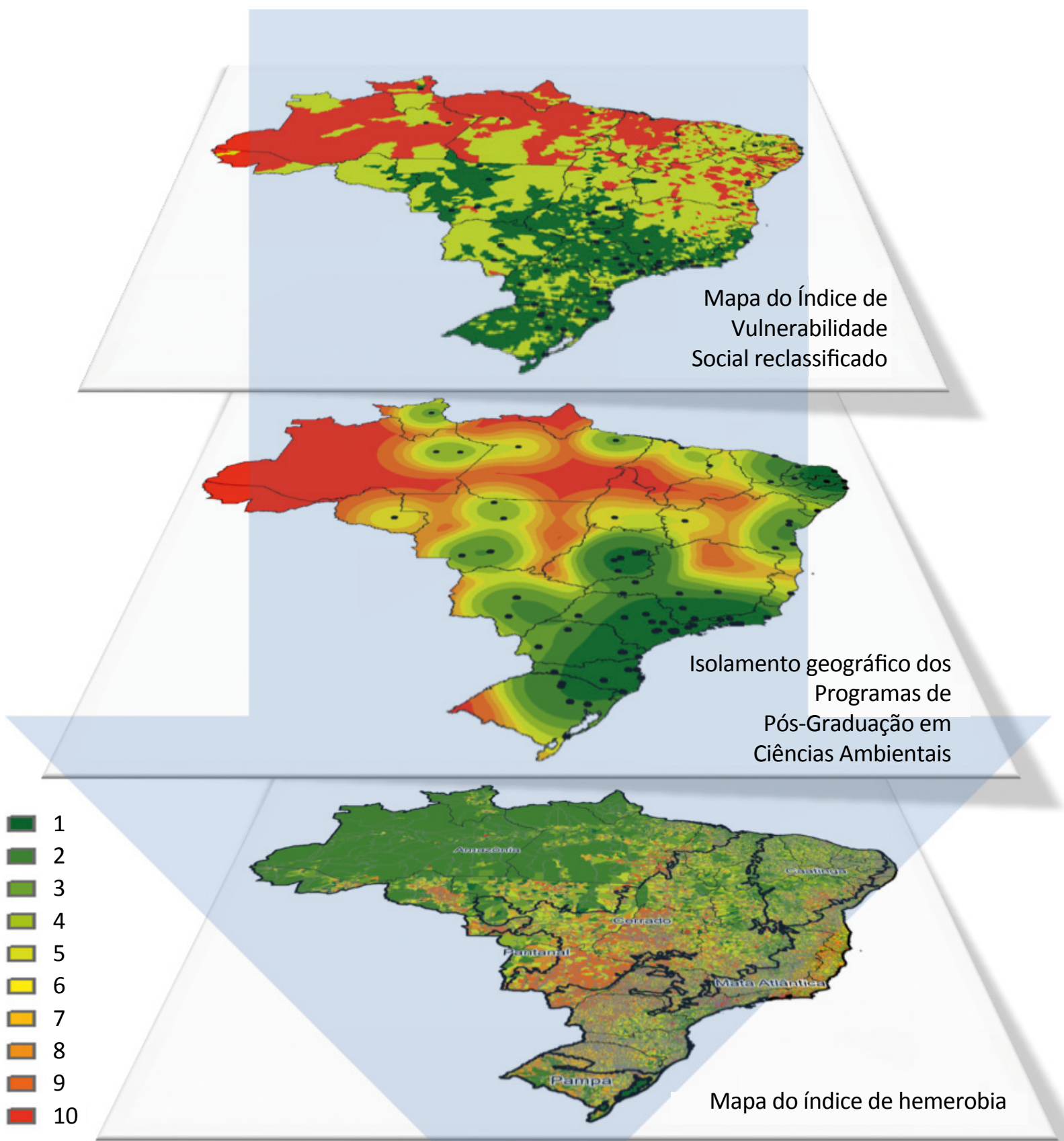

Figura 4 - Mapas resultantes dos componentes socioeconômico, socioambiental e espacial para a composição do modelo multicritério. 
A

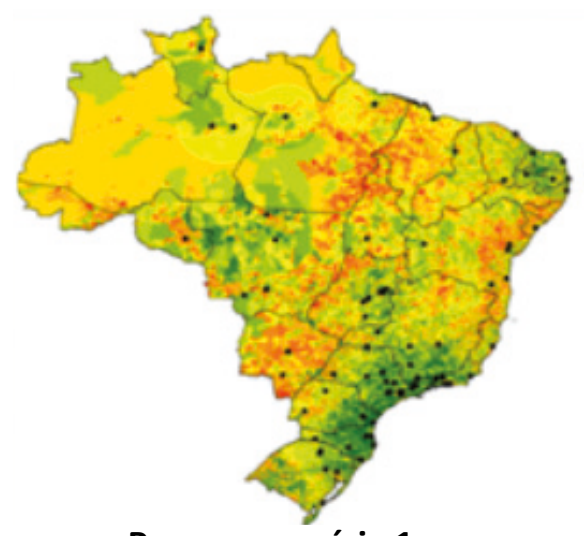

Pesos no cenário 1:

$50 \%$ socioambiental, $25 \%$ socioeconômico e $25 \%$ isolamento geográfico

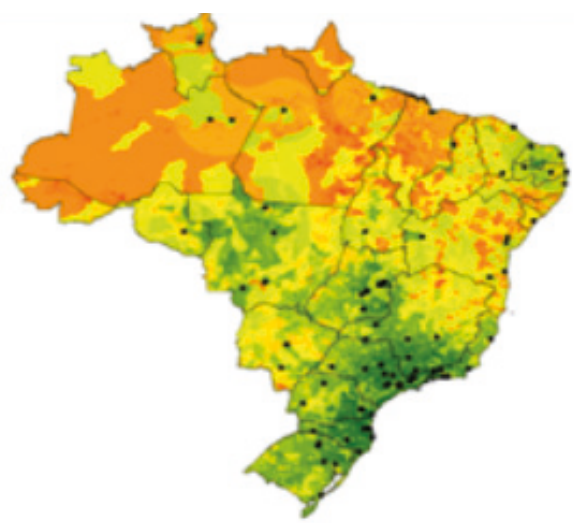

Pesos no cenário 2:

$25 \%$ socioambiental, $50 \%$ socioeconômico e $25 \%$ isolamento geográfico

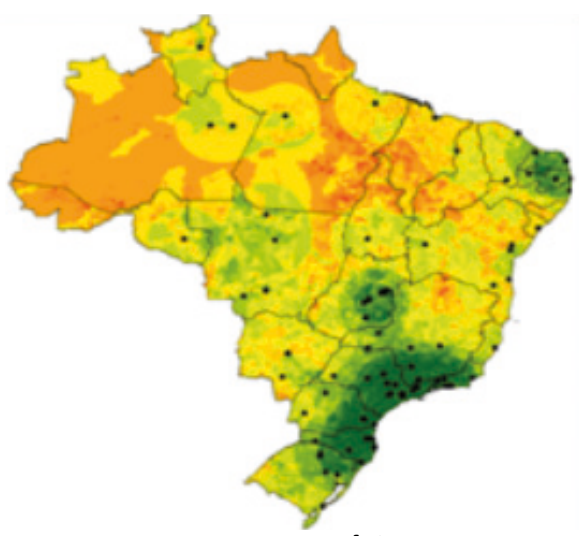

Pesos no cenário 3:

$25 \%$ socioambiental,

$25 \%$ socioeconômico e

$50 \%$ isolamento geográfico

B

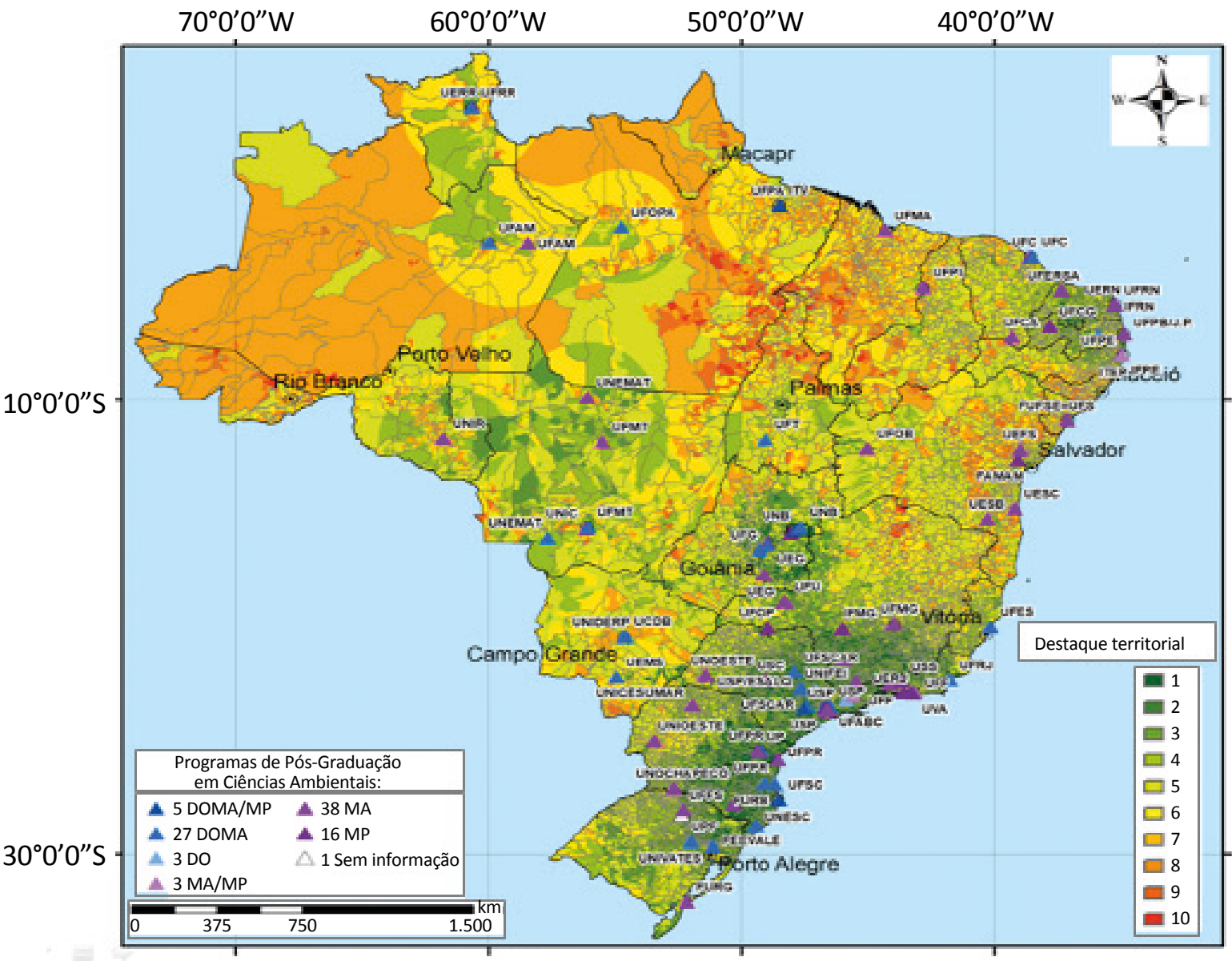

A. Cenários 1, 2 e 3: pesos diferenciados entre os componentes socioambiental, socioeconômico e isolamento geográfico.

B. Cenário 4: pesos iguais entre os componentes socioambiental, socioeconômico e isolamento geográfico.

(DOMA: doutorado e mestrado acadêmico; DO: doutorado; MA: mestrado acadêmico; MP: mestrado profissional).

Figura 5 - Mapas de destaque territorial com diferentes pesos atribuídos às dimensões socioeconômica, socioambiental e geográfica. 
paisagem revelado pela hemerobia. No cenário 2, predomina o critério socioeconômico e, portanto, as regiões de IVS mais acentuados contribuíram fortemente para elevar o índice de destaque territorial, particularmente na bacia amazônica. No cenário 3, prevalece o índice de isolamento geográfico dos PPG nas Ciências Ambientais e, nesse caso, nota-se que as regiões com concentração significativa de PPG possuem baixos índices de destaque territorial, ao passo que aquelas isoladas possuem valores mais elevados (Figura 5A).
Para minimizar discrepâncias decorrentes dos pesos atribuídos aos cenários 1,2 e 3, adotou-se um quarto cenário (Figura $5 \mathrm{~B}$ ), no qual os três critérios obtivessem o mesmo grau de influência no resultado (pesos iguais). Como implicação, o mapa de destaque territorial cumpriu o papel de revelar demandas por investimentos e ações de fomento na perspectiva da coordenação da área de Ciências Ambientais da CAPES. Além disso, ele abrange toda a extensão territorial brasileira, com riqueza de detalhe em nível submunicipal.

\section{Aplicação do mapa de destaque territorial na tomada de decisão}

O modelo proposto foi elaborado para auxiliar, de forma qualitativa, a tomada de decisão da Comissão de Avaliação Quadrienal 2017 dos PPG da área de Ciências Ambientais da CAPES durante o processo de avaliação quadrienal dos cursos de pós-graduação. Além dessa aplicação, o modelo também foi utilizado em uma análise quantitativa para apoiar a alocação de bolsas de estudo do Colegiado do PPG-AMSA da UFMG.

\section{Análise qualitativa: apoio na avaliação quadrienal 2017}

A Comissão de Avaliação Quadrienal 2017 dos PPG em Ciências Ambientais da CAPES utilizou o mapa de destaque territorial (Figura 5B) para conferir ao quesito Inserção Social uma métrica com interpretação qualitativa. Na ocasião, os consultores tiveram acesso ao mapa digital (cenário 4), com dez faixas de vulnerabilidade em escala de cores, e receberam também um roteiro utilizado na análise. Entre as classes 1 e 3 (predominância verde), considerou-se baixa vulnerabilidade socioeconômica e ambiental, de 4 a 7 (predominância amarela), média vulnerabilidade e de 8 a 10 (predominância vermelha), alta vulnerabilidade (Figura 5B).

A primeira etapa da análise de destaque territorial fazia referência à localização do PPG em relação ao município (capital, interior ou área de fronteira) e à espacialidade territorial em sua vizinhança, para identificar vulnerabilidades ou não da microrregião (estado e bioma). Na segunda etapa, o consultor identificava se o PPG estava inserido em área de vulnerabilidade, conforme as classes identificadas no mapa. Por último, uma análise qualitativa relacionava as informações descritas pelos PPG (projetos de pesquisa, teses ou dissertações, produções geradas, origem e perfil do discente/egresso) com o enfrentamento das vulnerabilidades socioeconômicas e socioambientais, sobretudo territoriais e microrregionais. A análise de destaque territorial considerou ainda a existência de PPG que poderiam contribuir com ações de enfrentamento de vulnerabilidades, ainda que não estejam fisicamente localizados em área de vulnerabilidade, utilizando-se informações como origem do discente, projetos de pesquisa, etc.

\section{Análise quantitativa: alocação de bolsa de estudo no Programa de Pós-Graduação em Análise e Modelagem de Sistemas Ambientais da Universidade Federal de Minas Gerais}

Nos últimos anos, a coordenação do Instituto de Geociências e do PPG-AMSA/UFMG vem incorporando ações de fortalecimento e inserção do programa nos cenários nacional e internacional. Uma das ações é a chamada nucleação, que estimula a captação de estudantes de pós-graduação oriundos de áreas distantes da Região Metropolitana de Belo Horizonte, onde o curso é sediado.
O objetivo do PPG-AMSA/UFMG é que as ações de nucleação possam atrair profissionais residentes preferencialmente em regiões com baixa oferta de vagas em cursos de pós-graduação em Ciências Ambientais e que a formação oferecida aos discentes possibilite o preenchimento de lacunas e a disseminação do conhecimento quando do retorno desses profissionais para suas regiões de origem. Nesse sentido, em seu último processo 
seletivo, o programa designou, em caráter singular, uma bolsa de estudos destinada a promover a nucleação. 0 critério de seleção do bolsista baseou-se na métrica de localização do município do candidato no mapa de destaque territorial, considerando-se os mesmos critérios do modelo proposto neste estudo (vulnerabilidade socioeconômica, ambiental e isolamento geográfico).

Três candidatos se inscreveram para a bolsa de nucleação, dois oriundos de municípios mineiros (Governador Valadares e Uberaba) e o outro, do Estado da Bahia (Capim Grosso). Após o lançamento dos polígonos dos municípios de origem dos candidatos sobre o mapa de destaque territorial, calculou-se o índice de vulnerabilidade de cada município, admitindo-se o valor médio do território para a tomada de decisão. Quanto maior o índice, maior o destaque territorial da região para ações em prol do fortalecimento da ciência e da educação. Após consulta ao mapa de destaque territorial, a bolsa de nucleação foi destinada ao candidato oriundo do município de Capim Grosso (BA), por apresentar o maior índice de vulnerabilidade dentre os municípios de origem dos discentes que participaram do processo seletivo (Figura 6).

A seleção do bolsista transcorreu de forma serena, objetiva e transparente, com pleno apoio da Pró-Reitoria de Pós-Graduação da UFMG.

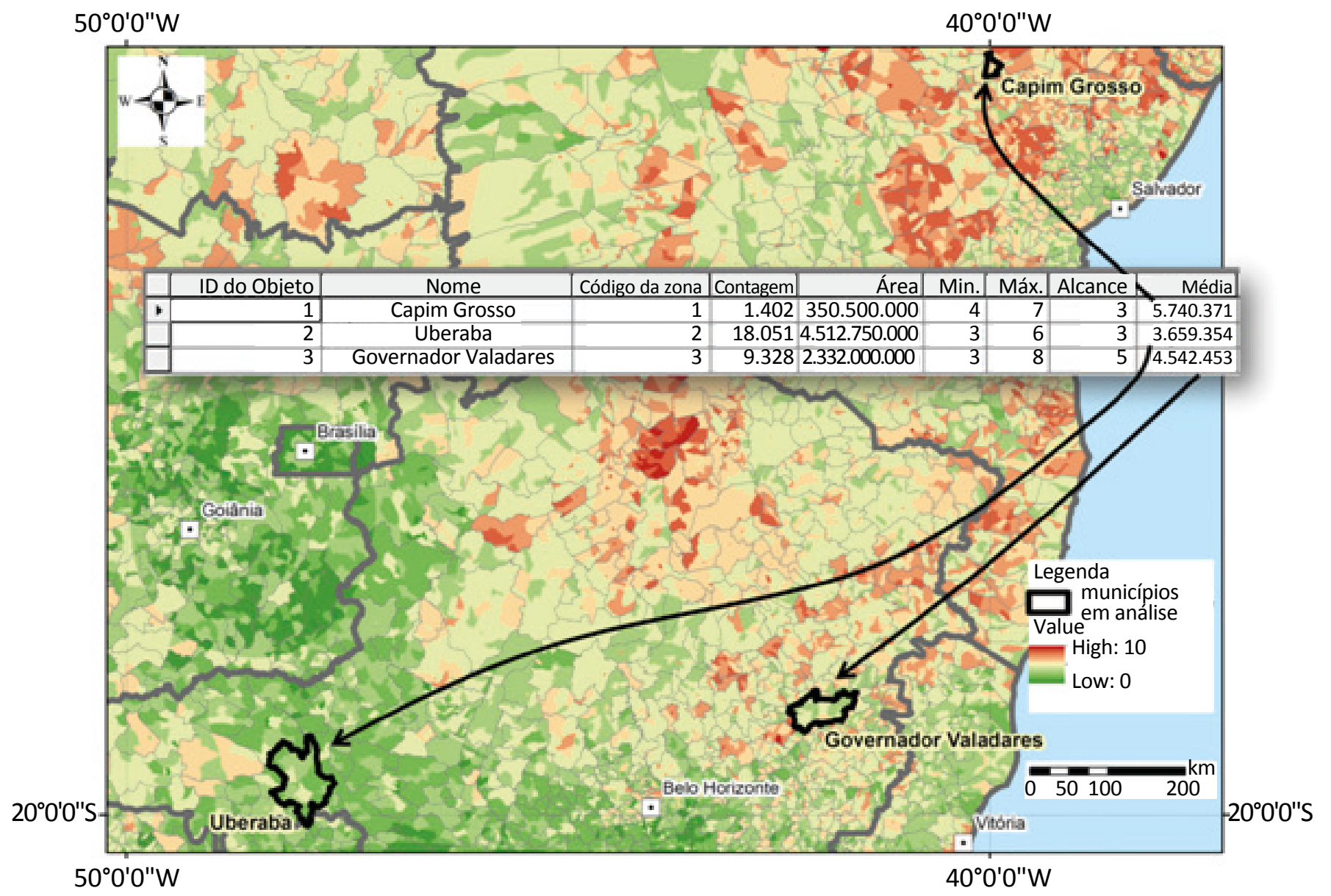

Figura 6 - Recorte geográfico do mapa de destaque territorial aplicado na seleção de candidato à bolsa de nucleação do Programa de Pós-Graduação Análise e Modelagem de Sistemas Ambientais da Universidade Federal de Minas Gerais para os ingressantes em 2018. 


\section{CONSIDERAÇÕES FINAIS}

Este artigo propõe uma metodologia para qualificar a inserção social dos PPG na área de Ciências Ambientais da CAPES, por meio da verificação do grau de vulnerabilidade socioeconômica e ambiental, designada ecossocioeconômica, dos municípios brasileiros e da utilização de técnicas de geotecnologia (modelagem espacial).

O modelo proposto integra três dimensões constituídas por critérios consolidados e robustos de análise territorial:

- socioeconômicos (IVS dos municípios brasileiros, elaborado pelo -IPEA);

- socioambientais (hemerobia calculada a partir dos dados de cobertura e de uso e ocupação do solo, disponibilizados pelo IBGE);

- isolamento geográfico (densidade da localização dos PPG em Ciências Ambientais).

Como resultado do modelo, tem-se o mapa de destaque territorial (escala nacional e detalhamento em nível municipal), que possibilita a visualização espacial dos PPG, bem como a identificação de vulnerabilidades regionais e do potencial de contribuição dos PPGs em suas áreas de influência.

Para validação do modelo, utilizou-se o mapa de destaque territorial para apoiar a tomada de decisão em duas oportunidades distintas. A primeira, no processo de avaliação quadrienal 2017 da área de Ciências Ambientais da CAPES, em relação à inserção social dos PPG (análise qualitativa), e a segunda na seleção de um candidato à bolsa de estudo do PPG-AMSA/UFMG, para a nucleação de estudantes procedentes de áreas em condição de vulnerabilidade socioeconômica e ambiental (análise quantitativa). Nos dois casos, obteve-se o êxito almejado.
Além das aplicações examinadas neste estudo, outras possibilidades de utilização do mapa de destaque territorial da CAPES são vislumbradas pela coordenação da área de Ciências Ambientais: diagnóstico de localidades prioritárias para indução de novos cursos; direcionamento de talentos humanos e recursos financeiros em editais destinados a áreas em condições de vulnerabilidade socioeconômica e ambiental; incentivo à criação de novas redes de pesquisa e de colaboração entre PPG inseridos em áreas mais vulneráveis e menos vulneráveis; mapeamento das atuações e dos impactos das pesquisas, do ensino e da extensão dos PPGs (âmbitos local, regional e nacional); e diagnóstico da inserção profissional dos egressos dos PPG (sobretudo os discentes procedentes de áreas vulneráveis).

Nesse sentido, a modelagem proposta constitui-se em uma metodologia inovadora, capaz de ampliar a capacidade de análise da área de Ciências Ambientais da CAPES, por meio da espacialização de critérios múltiplos, e inédita, pois não há literatura semelhante.

Embora o modelo apresentado seja robusto (capacidade de processamento e resultados) e flexível (possibilidade de adaptação e reprodutividade), os autores constataram a necessidade de inclusão de novos dados que possam apurar o levantamento das demandas regionais, seja pela qualidade da informação, seja pela sua atualização. Nesse sentido, identificou-se potencial para o aperfeiçoamento do modelo por meio do emprego de informações de cobertura do solo provenientes do Projeto MapBiomas (dados mais recentes e com maior resolução espacial). De forma análoga, uma alternativa a ser considerada para o componente de isolamento geográfico consiste na integração de outros dados, como distância entre o PPG e as agências de fomento e de capitais, a densidade de rodovias e o distanciamento de aeroportos, entre outros critérios.

\section{AGRADECIMENTOS}

Os autores agradecem à coordenação da área de Ciências Ambientais da CAPES o apoio, ao IBGE e ao
IPEA o fornecimento dos dados utilizados no trabalho, bem como ao CNPq o suporte à pesquisa. 


\section{REFERÊNCIAS}

AGÊNCIA AMBIENTAL EUROPEIA (AAE). Programa CORINE Land Cover. Europa: AAE, 2018. Disponível em: <www.eea. europa.eu/publications/CORO-landcover>. Acesso em: 31 jul. 2018.

BELEM, A. L. G.; NUCCI, J. C. Hemerobia das paisagens: conceito, classificação e aplicação no bairro Pici - Fortaleza/CE. Raega, v. 21, p. 204-233, 2011.

BRASIL. Coordenação de Aperfeiçoamento de Pessoal de Nível Superior (CAPES). Coleta Capes-Dados cadastrais dos programas. Plataforma Sucupira. Brasil: CAPES, 2018. Disponível em: <https://sucupira.capes.gov.br/sucupira/public/consultas/coleta/ programa/listaPrograma.jsf;jsessionid=MEwq3XMIVqDjTKwUj2XpjBC+.sucupira-2182018>. Acesso em: 26 jun. 2018.

. Coordenação de Aperfeiçoamento de Pessoal de Nível Superior (CAPES). Plano Nacional de Pós-Graduação (PNPG) 2011-2020. Documentos Setoriais. Brasília: CAPES, 2010. v. 2. Disponível em: <www.capes.gov.br/images/ stories/download/PNPG_Miolo_V2.pdf>. Acesso em: 27 jun. 2018.

BURROUGH, P.; MCDONNELL, R. Principles of Geographic Information Systems. Nova York: Oxford University Press, 1998. $333 \mathrm{p}$.

CÂMARA, G.; MONTEIRO, A. M. V.; DRUCK, S.; CARVALHO, M. S. Análise espacial e geoprocessamento. In: DRUCKS, S.; CARVALHO M. S.; CÂMARA, G.; MONTEIRO, A. M. V. (Eds.). Análise espacial de dados geográficos. EMBRAPA, 2004.

CARVALHO-RIBEIRO, S.; PINTO CORREIA, T.; PARACCHINI, M. L.; SCHÜPBACH, B.; ODE SANG, A.; VANDERHEYDEN, V.; SOUTHERN, A.; JONES, P.; CONTRERAS, B.; O'RIORDAN, T. Assessing the ability of rural agrarian areas to provide cultural ecosystem services (CES): A multi scale social indicator framework (MSIF). Land Use Policy, v. 53, p. 8-19, 2016. http:// dx.doi.org/10.1016/j.landusepol.2015.04.024

DIAS SOBRINHO, J. Avaliação ética e política em função da educação como direito público ou como mercadoria? Educação \& Sociedade, v. 25, n. 88, p. 703-725, 2004. Disponível em: <www.scielo.br/pdf/es/v25n88/a04v2588.pdf>. Acesso em: 27 jun. 2018.

DONOVAN, C. The Australian Research Quality Framework: A live experiment in capturing the social, economic, environmental and cultural returns of publicly funded research. In: CORYN, C. L. S.; SCRIVEN, M. (Orgs.). Reforming the Evaluation of Research. New Directions for Evaluation, v. 118, p. 47-60, 2008.

ESTOQUE, R. C.; MURAYAMA, Y. A worldwide country-based assessment of social-ecological status using the socialecological status index. Ecological Indicators, v. 72, p. 605-614, 2017. https://doi.org/10.1016/j.ecolind.2016.08.047

EUROPEAN COMMISSION. European Research Area. Assessing Europe's University-Based Research: Expert Group on Assessment of University-Based Research. Bruxelas: European Commission, 2010. Disponível em: <https://ec.europa. eu/research/science-society/document_library/pdf_06/assessing-europe-university-based-research_en.pdf>. Acesso em: 28 jun. 2018.

FERNANDES, V.; SAMPAIO, C. A. C. A natureza da problemática socioambiental. In: SILVA, S. D.; SAYAGO, D.; TONI, F; CAMPOS, F. I. Ensaios em ciências ambientais: crises riscos e racionalidades. Rio de Janeiro: Garamond, 2016. v. 1. p. 153-166.

FERRAZ, C. A. M.; VIEIRA, R. R. T.; BERBERIAN, C. F. Q.; DIAS FILHO, N.; NÓBREGA, R. A. A. O uso de geotecnologias como uma ferramenta para o Controle Externo. Revista do Tribunal de Contas da União, v. 133, p. 40-53, 2015.

FERREIRA JUNIOR, J. I.; NOBREGA, R. A. A.; OLIVEIRA, L. K. Geographic data modeling to define alternative transport corridors to bypass the Metropolitan Region of Belo Horizonte: comparative scenarios. Revista do Tribunal de Contas da União, v. 137, p. 1-10, 2016. 
FURTADO, A. T.; BIN, A.; BONACELLI, M. B. M.; PAULINO, S. R.; MIGLINO, M. A.; CASTRO, P. F. D. Evaluation of the results and impacts of a social-oriented technology program in Brazil: the case of PROSAB (a sanitation research program). Research Evaluation, v. 18, n. 4, p. 289-300, 2009. https://doi.org/10.3152/095820209X451014

FUSHITA, A. T. Padrão espacial e temporal das mudanças de uso da terra e sua relação com indicadores da paisagem. Estudo de caso: bacia hidrográfica do médio rio Mogi-Guaçu superior/SP. $228 \mathrm{f}$. Tese (Doutorado em Ecologia e Recursos Naturais)-Universidade Federal de São Carlos, São Carlos, 2011.

FUSHITA, A. T.; SANTOS, J. E.; ROCHA, Y. T.; ZANIN, E. M. Historical Land Use / Cover Changes and the Hemeroby Levels of a Bio-Cultural Landscape: Past, Present and Future. Journal of Geographic Information System, v. 9, n. 5, p. 576-590, 2017. https://doi.org/10.4236/jgis.2017.95036

HAFFER, J. Ciclos de tempo e indicadores de tempos na história da Amazônia. Estudos Avançados [conectados], v. 6, n. 15, p. 7-39, 1992. Disponível em: <http://www.scielo.br/scielo.php?script=sci_arttext\&pid=S0103-40141992000200002>. Acesso em: 31 ago. 2018. http://dx.doi.org/10.1590/S0103-40141992000200002

HUMMELL, B. M. L.; CUTTER, S. L.; EMRICH, C. T. Social Vulnerability to Natural Hazards in Brazil. International Journal of Disaster Risk Science, v. 7, n. 2, p. 111-122, 2016. https://doi.org/10.1007/s13753-016-0090-9

INSTITUTO BRASILEIRO DE GEOGRAFIA E ESTATÍSTICA (IBGE). Monitoramento da cobertura e uso da terra do Brasil. IBGE, 2012. Disponível em: <www.ibge.gov.br/apps/monitoramento_cobertura_uso_terra/v1/>. Acesso em: 26 jun. 2018.

INSTITUTO DE PESQUISA ECONÔMICA APLICADA (IPEA). Atlas da vulnerabilidade social nos municípios brasileiros. Brasília: IPEA, 2015. Disponível em: <http://ivs.ipea.gov.br/images/publicacoes/Ivs/publicacao_atlas_ivs_rm.pdf>. Acesso em: 26 jun. 2018.

INTERNATIONAL ASSOCIATION LANDSCAPE ECOLOGY (IALE). Boletim do Simpósio. IALE, 1998. Disponível em: <www. landscape-ecology.org/fileadmin/user_upload/content/bulletin/bulletin16_6e.pdf>. Acesso em: 10 ago. 2018.

KRAKER, J. Social learning for resilience in social-ecological systems. Current Opinion in Environmental Sustainability, v. 28, p. 100-107, 2017. https://doi.org/10.1016/j.cosust.2017.09.002

LANG, S.; BLASCHKE, T. Análise da paisagem com SIG. São Paulo: Oficina de Textos, 2009.

MALCZEWSKI, J. GIS and Multi-criteria Decision Analysis. Nova York, Wiley, 1999.

NOBREGA, R. A. A. Geoprocessamento: a importância de conhecer o onde, o quando e o quanto no planejamento e gestão territorial e na definição de políticas públicas. Fonte, Belo Horizonte, v. 15, n. 19, p. 62-63, 2018.

. Understanding Spatial-Criteria Decision Making: an analytical demonstration of AHP-based MCDM and how it is used in GIS. In: NATIONAL CONSORTIUM FOR REMOTE SENSING AND TRANSPORTATION ANNUAL MEETING, Memphis. Memphis Workshop. Memphis: National Consortium for Remote Sensing and Transportation Annual Meeting, 2009.

NOBREGA, R. A. A.; BROOKS, C.; OHARA, C. G.; STICH, B. Multi-scale GIS Data-driven Method for Early Assessment of Wetlands Impacted by Transportation Corridors. In: ALAM, B. M. (Org.). The Geographic Information System. Rijeka: InTech, 2012. p. 20.

O'HARA, C. G.; DAVIS, A. A.; KLEISS B. A. A decision support system for prioritizing forested wetland restoration in the Yazoo Backwater Area, Mississippi, working paper. Water Resources Investigation Report 00-4199. Pearl, MS: United States Geological Service, 2000.

PENFIELD, T.; BAKER, M. J.; SCOBLE, R.; WYKES, M. C. Assessment, evaluations, and definitions of research impact: A review. Research Evaluation, v. 23, n. 1, p. 21-32, 2014. https://doi.org/10.1093/reseval/rvt021 
RESEARCH EXCELLENCE FRAMEWORK (REF). Department for Employment and Learning. Decisions on assessing research impact. Reino Unido: REF, 2014. Disponível em: <www.ref.ac.uk/2014/media/ref/content/pub/ decisionsonassessingresearchimpact/01_11.pdf>. Acesso em: 28 jun. 2018.

RÜDISSER, J.; TASSER, E.; TAPPEINER, U. Distance to nature - A new biodiversity relevant environmental indicator set at the landscape level. Ecological Indicators, v. 15, n. 1, p. 208-216, 2012. http://doi.org/10.1016/j.ecolind.2011.09.027

RYERSON, B.; ARONOFF, S. Why 'Where' Matters: Understanding and Profiting from GPS, GIS, and Remote Sensing. Manotik: Kim Geomatics Corporation, 2010. 379 p.

SAATY, T. L. Fundamentals of Decision Making and Priority Theory with the AHP. Petersburgo: RWS Publications, 1994.

SADASIVUNI, R.; NOBREGA, R. A. A.; OHARA, C. G.; DUMAS, J. A Transportation Corridor Case Study for Multi-Criteria Decision Analysis. In: AMERICAN SOCIETY OF PHOTOGRAMMETRY AND REMOTE SENSING ANNUAL MEETING, 2009, Baltimore. Proceedings of 75th ASPRS annual meeting. Baltimore, 2009.

SAMPAIO, C. A. C.; CORTEZ, J. S. A.; SCHIMITT, J. L. Documento de área - Ciências Ambientais. Brasília: CAPES, 2016. Disponível em: <www.capes.gov.br/images/documentos/Documentos_de_area_2017/49_CAMB_docarea_2016_ publ2.pdf>. Acesso em: 26 jun. 2018.

SAMPAIO, C. A. C.; CORTEZ, J. S. A.; SCHIMITT, J. L. Relatório de Avaliação Quadrienal - Ciências Ambientais. Brasília: CAPES, 2017. Disponível em: <www.capes.gov.br/images/stories/download/avaliacao/relatorios-finais-quadrienal2017/20122017-CIENCIAS-AMBIENTAIS-quadrienal.pdf>. Acesso em: 26 jun. 2018.

SOBRAL, M. C. M.; SAMPAIO, C. A. C.; FERNANDES, V.; PHILIPPI JR., A. Práticas interdisciplinares em ciências ambientais. In: PHILIPPI JR., A.; FERNANDES, V. Práticas da interdisciplinaridade no ensino e pesquisa. Barueri: Manole, 2014.

SPAGNOLO, F.; SOUZA, V. C. O que mudar na avaliação da CAPES? Revista Brasileira de Pós-graduação, v. 1, n. 2, p. 8-34, 2004. http://dx.doi.org/10.21713/2358-2332.2004.v1.38

SPAAPEN, J.; DROOGE, L. Introducing 'productive interactions' in social impact assessment. Research Evaluation , v. 20, n. 3, 2011, p. 211-218.

STICH, B.; HOLLAND, J. H.; NOBREGA, R. A. A.; O'HARA C. G. Using multi-criteria decision making to highlight stakeholders values in the corridor planning process. Journal of Transport and Land Use, v. 4, n. 3, p. 105-118, 2011. http://dx.doi. org/10.5198/jtlu.v4i3.171

SUKOPP, H. Wandel von Flora und Vegetation in Mitteleuropa unter dem Einfluss des Menschen. Berichte uber Landwirtschaft, 50/H.1, p. 112-139, 1972.

TROPPMAIR, H. Biogeografia e Meio Ambiente. Rio Claro: Technical Books, 1989. 258 p.

WALZ, U.; STEIN, C. Indicators of hemeroby for the monitoring of landscapes in Germany. Journal for Nature Conservation, v. 22, n. 3, p. 279-289, 2014. https://doi.org/10.1016/j.jnc.2014.01.007

WELLMANN, T.; HAASE, D.; KNAPP, S.; SALBACH, C.; SELSAM, P.; LAUSCH, A. Urban land use intensity assessment: The potential of spatio-temporal spectral traits with remote sensing. Ecological Indicators, v. 85, p. 190-203, 2018. https:// doi.org/10.1016/j.ecolind.2017.10.029 